\title{
La peatonabilidad desde la perspectiva sistémico- sustentable y la calidad de la accesibilidad radial del Parque Central de Ciudad Juárez, México
}

Pedestrianization from the systemic-sustainable perspective and the quality of radial accessibility of Parque Central in Juárez, México

A pedestre desde a perspectiva sistêmico-sustentável e a qualidade da acessibilidade radial do Parque Central de Ciudad Juárez, México

Diego Adiel Sandoval Chávez

Tecnológico Nacional de México, Instituto Tecnológico de Ciudad Juárez, México dsandoval@itcj.edu.mx https://orcid.org/0000-0002-2536-1844

Aida Yarira Reyes Escalante Universidad Autónoma de Ciudad Juárez, México aida.reyes@uacj.mx https://orcid.org/0000-0002-0104-9522

Ana Córdova y Vázquez

El Colegio de la Frontera Norte, México acordova@colef.mx https://orcid.org/0000-0003-4551-5123

Luz Elena Tarango Hernández Tecnológico Nacional de México, Instituto Tecnológico de Ciudad Juárez, México Itarango@itcj.edu.mx https://orcid.org/0000-0003-4194-9709 


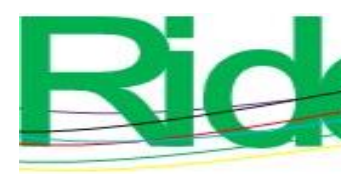

Revista Iberoamericana para la
Investigación y el Desarrollo Educativo
ISSN $2007-7467$

Luz Elena Terrazas Mata

Tecnológico Nacional de México, Instituto Tecnológico de Ciudad Juárez, México

Iterrazas@itcj.edu.mx

https://orcid.org/0000-0003-1396-1751

\section{Resumen}

La peatonabilidad es una característica de las ciudades sustentables. En el caso de Ciudad Juárez, México, se tienen serios rezagos en infraestructura urbana y malas prácticas cívicas que afectan el traslado a pie de las personas hacia el espacio público. El objetivo de este trabajo es evaluar la accesibilidad radial tomando como referencia un parque urbano. La investigación adopta un método de observación directa en las rutas radiales considerando a un adulto sano. El análisis de los datos se basó en una visión sistémica de distinción entre la variabilidad aleatoria y la variabilidad especial con base en la distribución de Poisson y un posterior análisis de criticidad. El estudio se realizó en el entorno construido a un radio de $800 \mathrm{~m}$ del Parque Central ubicado en Ciudad Juárez. Se evaluaron seis rutas identificando y registrando los obstáculos fijos y no fijos que impedían o restringían el tránsito caminando. Los resultados revelaron que prevalecen cinco obstáculos de alta criticidad que afectan a la peatonabilidad y, por consiguiente, a la calidad de la accesibilidad radial del parque. Tres de estos obstáculos se asociaron con el estado de las aceras y los dos restantes con el estado de las calles. Se analizan escenarios de gestión para abordar esta problemática y se discute su relevancia. Finalmente, se concluye que el enfoque utilizado es pertinente para el estudio de la calidad de la accesibilidad radial del parque y se sugiere una agenda de investigación para futuros trabajos.

Palabras clave: caminabilidad, entorno construido, entropía urbana, parque urbano, sustentabilidad urbana. 


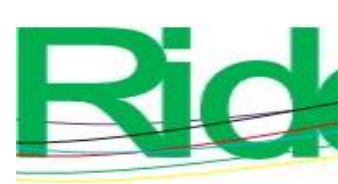

Revista Iberoamericana para la Investigación y el Desarrollo Educativo ISSN $2007-7467$

\section{Abstract}

Pedestrianization is a key factor for sustainable cities. In the case of Ciudad Juarez, Mexico, there are serious shortcomings in urban infrastructure and poor civic practices that affect the mobility of people on foot to public space. The objective of this work is to evaluate radial accessibility taking an urban park as a reference. The research adopts a direct observation method on radial routes considering a healthy adult. The data analysis was based on a systemic view that distinguishes between random variability and assignable variability adopting Poisson distribution and a subsequent criticality analysis. The study was conducted in the built environment within an $800 \mathrm{~m}$ radius of Parque Central located in Juarez. Six routes were evaluated by identifying and recording fixed and non-fixed obstacles that impeded or restricted walking traffic. Results revealed the prevalence of five high criticality obstacles affecting pedestrian walkability and, consequently, the quality of radial accessibility of the park. Three of these obstacles were associated with the condition of the sidewalks and the remaining two with the condition of the streets. Management scenarios to address this issue are analyzed and their relevance is discussed. Finally, it is concluded that the approach used is relevant to the study of the quality of radial accessibility of the park and a research agenda for future work is suggested.

Keywords: walkability, built environment, urban entropy, urban park, urban sustainability.

\section{Resumo}

A caminhabilidade é uma característica das cidades sustentáveis. No caso de Ciudad Juárez, no México, há sérios atrasos na infraestrutura urbana e más práticas cívicas que afetam o deslocamento de pessoas para os espaços públicos a pé. O objetivo deste trabalho é avaliar a acessibilidade radial tomando como referência um parque urbano. A pesquisa adota um método de observação direta nas rotas radiais considerando um adulto saudável. A análise dos dados foi baseada em uma visão sistêmica de distinção entre variabilidade aleatória e variabilidade especial com base na distribuição de Poisson e posterior análise de criticidade. O estudo foi realizado no ambiente construído em um raio de $800 \mathrm{~m}$ do Central Park localizado em Ciudad Juárez. Seis rotas foram avaliadas identificando e registrando obstáculos fixos e não fixos que impediam ou restringiam o tráfego de pedestres. Os resultados revelaram que prevalecem cinco obstáculos altamente críticos que afetam os 


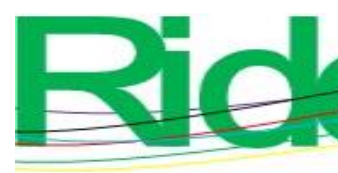

Revista Iberoamericana para la Investigación y el Desarrollo Educativo ISSN 2007- 7467

espacios (Zuniga et al., 2019). La accesibilidad radial a los espacios de recreación, principalmente los parques urbanos, debe incluir un ambiente libre de dificultades que impidan el caminar hacia ellos. Un parámetro muy conocido se presenta desde la metáfora “espacio 8-80", que se refiere a que un espacio público es accesible si tanto un infante de ocho años como un adulto mayor de 80 años pueden acceder a pie sin obstáculos que se los impida (Peñalosa, 2018).

Las dimensiones más referidas de la caminabilidad son: Conectividad, Conveniencia, Confortabilidad, Convivencia y Conspicuidad, a las cuales suele agregárseles Coexistencia y Compromiso, referidas como las 7C (Moura, Cambra y Gonçalves, 2017) (tabla 1). Su estudio se aborda desde las disciplinas de la ingeniería o la arquitectura, considerando enfoques basados en la observación directa (listas de auditoría y verificación, inventarios, niveles de servicio, escalas de valoración) y otras herramientas como la encuesta o la entrevista, además del uso de sistemas de información geográfica (SIG) (Moura et al., 2017; Shashank y Schuurman, 2019; Talavera y Soria, 2015; Telega, Telega y Bieda, 2021).

Una constante en las investigaciones de caminabilidad es su temporalidad transversal: se estudia en una sola medición el entorno construido y se reporta, según el enfoque adoptado, el estado o grado de caminabilidad que guarda el espacio estudiado (Maghelal y Capp, 2011). Si bien este enfoque es práctico y pertinente, no fue posible identificar estudios que consideren la naturaleza sistémica de la ciudad y que reconozcan que el desarrollo urbano, más que una acción aislada, es un proceso que guarda continuidad y se configura de conformidad con la ejecución de las acciones en las ciudades desde diferentes visiones e ideologías, con intensa interacción, las cuales delinean el perfil de la ciudad e influyen en sus prácticas democráticas (Pinson y Morel, 2016). Esto es: la ciudad es un sistema y como tal es posible ubicarla en el nicho epistemológico de la teoría general de sistemas, siguiendo sus principios y características (von Bertalanffy, 1976). 


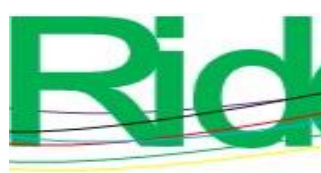

Revista Iberoamericana para la
Investigación y el Desarrollo Educativo
ISSN $2007-7467$

En Ciudad Juárez, se tienen serios rezagos en infraestructura urbana y malas prácticas cívicas que afectan el traslado a pie de las personas hacia el espacio público. Para el caso del Parque Central, se desconoce la calidad de la accesibilidad radial en su radio de influencia y las estrategias de gestión pertinente para mejorarla. Con base en lo anteriormente expuesto, el objetivo de este trabajo es evaluar la accesibilidad radial tomando como referencia un parque urbano.

\section{Método}

El Parque Central de Ciudad Juárez, México, que anualmente recibe entre 1100 0001300000 visitantes, fue el sitio donde se llevó a cabo el estudio. El espacio comprende una superficie de 20 ha de áreas verdes y cuerpos de agua. El parque se sitúa a $31.73^{\circ}$ latitud norte y $106.48^{\circ}$ longitud oeste (figura 1). Su oferta incluye instalaciones de recreación, ejercicio, corredores de vegetación, jardín botánico y varias plazuelas para realizar actividades pasivas o de relajación. El parque cuenta con un lago artificial que imprime belleza paisajística y promueve la diversidad; el lago alberga peces (carpas, truchas y bagres), dos especies de tortugas, además de ser utilizado por aves de diversas especies, principalmente patos. Uno de sus mayores atractivos es una jirafa macho, que se ha convertido en el símbolo del parque.

Para evaluar la accesibilidad radial, se eligieron seis rutas al azar en un radio de 800 $\mathrm{m}$ alrededor del parque (figura 2), las cuales se ajustaron a la lógica del trayecto en dirección al parque, esto es, buscando la ruta más corta y sin rodeos innecesarios; el tiempo de recorrido de una determinada ruta se estima entre 10-15 min.

Figura 1. Aspectos del Parque Central en Ciudad Juárez, México

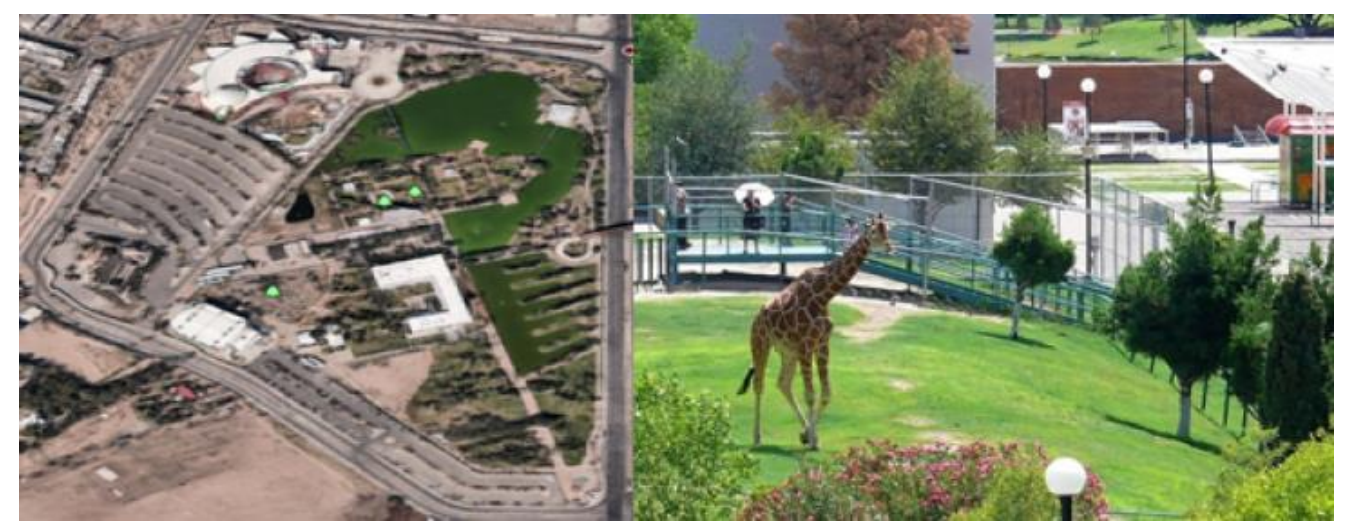

Fuente. Elaboración propia 
Tabla 2. Elementos de evaluación de la accesibilidad radial a $800 \mathrm{~m}$ del Parque Central

\begin{tabular}{|c|c|c|c|}
\hline \multicolumn{2}{|c|}{ Obstáculos fijos } & \multicolumn{2}{|r|}{ Obstáculos móviles } \\
\hline \multirow[t]{4}{*}{ Acera } & Faltante & \multirow[t]{4}{*}{ Acera } & Bloqueada por automóvil \\
\hline & En mal estado & & Bloqueada por material \\
\hline & $\begin{array}{l}\text { Bloqueada por elemento } \\
\text { fijo }\end{array}$ & & Bloqueada por anuncios \\
\hline & Demasiado estrecha & & Bloqueada por mercancía \\
\hline \multirow[t]{3}{*}{ Rampa } & Faltante & \multirow[t]{4}{*}{ Comercio } & Puesto comercial en acera \\
\hline & En mal estado & & Puesto comercial en calle \\
\hline & $\begin{array}{l}\text { Bloqueada por elemento } \\
\text { fijo }\end{array}$ & & $\begin{array}{l}\text { Mercado o vehículo } \\
\text { comercial }\end{array}$ \\
\hline \multirow[t]{3}{*}{ Pavimento } & Faltante & & Talleres mecánicos \\
\hline & En mal estado & \multirow[t]{5}{*}{ Vehículos } & Bloqueando calle \\
\hline & Suelto o baches & & En doble fila \\
\hline \multirow[t]{3}{*}{ Horadación } & Hoyo en acera & & Estacionados en batería \\
\hline & Hoyo en calle & & Abandonados \\
\hline & Zanja & & Vehículo de carga presente \\
\hline \multirow[t]{7}{*}{$\begin{array}{l}\text { Discontinuidad } \\
\text { en el trayecto }\end{array}$} & $\begin{array}{l}\text { Desvío obligado de ruta } \\
\text { lógica }\end{array}$ & \multirow[t]{10}{*}{ Diversos } & $\begin{array}{l}\text { Desechos orgánicos de } \\
\text { animales }\end{array}$ \\
\hline & $\begin{array}{l}\text { Puesto de seguridad } \\
\text { privada }\end{array}$ & & $\begin{array}{l}\text { Postes, árboles o ramas } \\
\text { tiradas }\end{array}$ \\
\hline & $\begin{array}{l}\text { Calle cerrada por } \\
\text { seguridad }\end{array}$ & & $\begin{array}{l}\text { Contenedores de basura, } \\
\text { macetas }\end{array}$ \\
\hline & Calle cerrada (otro motivo) & & Otros \\
\hline & Rejas o cerco salvable & & \\
\hline & Tope reductor de velocidad & & \\
\hline & Dren, acequia o arroyo & & \\
\hline \multirow[t]{2}{*}{ Avenida } & Avenida principal & & \\
\hline & Puente o paso peatonal & & \\
\hline Diversos & Otros & & \\
\hline
\end{tabular}

Fuente: Elaboración propia 


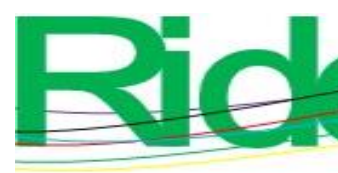

Revista Iberoamericana para la
Investigación y el Desarrollo Educativo
ISSN $2007-7467$

En este caso, la probabilidad de que un obstáculo esté presente en un trayecto determinado depende de la longitud del tramo recorrido. Asimismo, al considerar seis segmentos de igual magnitud en el mismo entorno urbano, se cuenta con una unidad de estudio uniforme. Ahora bien, los obstáculos se presentan con independencia entre las rutas estudiadas. Las anteriores consideraciones son características de los fenómenos que siguen la distribución de Poisson (Walpole, Myers, Myers y Ye, 2012). Por lo tanto, se tiene que la probabilidad de encontrar $k$ obstáculos en una determinada ruta está dada por:

$$
p(k, \lambda)=\frac{e^{-\lambda} \lambda^{k}}{k !} k=0,1,2, \ldots
$$

En dicha fórmula $\lambda$ es el parámetro de la distribución de Poisson. Entonces, el círculo que tiene como radio $800 \mathrm{~m}$ es el sistema a estudiar y la ruta $R_{i}$ es la entidad uniforme que se ve afectada por la variación natural o aleatoria, inherente al universo de obstáculos comprendidos dentro del círculo, y la variación especial o relativa, atribuible a los obstáculos específicos de la ruta $R i$ :

$$
S_{t_{i}}=S_{c}+S_{R_{i}} i=1,2,,,,, 6
$$

En la fórmula 2, por su parte:

- $\quad S_{t i}$ es la variación total de la ruta $i$.

- $\quad S_{c}$ es la variación aleatoria de la región considerada (círculo con radio de 800

$\mathrm{m})$.

- $\quad S_{R i}$ es la variación especial atribuible a la ruta $i$.

Ya que en la distribución de Poisson $E(x)=\lambda$ y $V(x)=\lambda$, el rango de $S_{c}$ es:

$$
S_{c}=\lambda \pm 3 \sqrt{\lambda}
$$

Este esquema reveló cuáles rutas presentaron un número de obstáculos mayor al rango de la variación aleatoria, lo que permitió conocer el tipo de obstáculos específicos de cada ruta.

\section{Resultados}

Los resultados de los recorridos de las rutas en cuanto a la frecuencia de obstáculos se ajustan a una distribución de Poisson $(p<0.320)$ y se muestran en la tabla 3. Las rutas uno, dos y tres se muestran en la figura 3 y las rutas cuatro, cinco y seis se muestran en la figura 4. Los obstáculos fijos con mayor frecuencia son: acera faltante, acera en mal estado, 

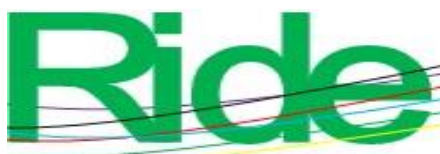

pavimento suelto o baches, acera bloqueada por elemento fijo y tope reductor de velocidad. Tres de ellos están relacionados con las aceras, un elemento crucial para la accesibilidad radial (Landin, 23 de febrero de 2016). La evaluación inicia analizando la frecuencia con la que se presentan en cada ruta; después, y a fin de discriminar entre la importancia de los obstáculos fijos para la accesibilidad radial, se incorporó en el análisis una ponderación ordinal de criticidad. Este análisis permitió identificar cuán accesible es cada ruta para el traslado al parque caminando.

La figura 5 muestra los resultados del procesamiento de los hallazgos mediante un gráfico basado en la distribución de Poisson, de acuerdo con la ecuación 1. El valor del parámetro $\lambda$ se estimó en 1.15 obstáculos/ruta. Entonces, de la ecuación 3, los límites inferior y superior de la variación aleatoria inherente a la región estudiada son:

$$
S_{C}=\lambda \pm 3 \sqrt{\lambda}=1.15 \pm 3 \sqrt{1.15}=(0,4.37)
$$




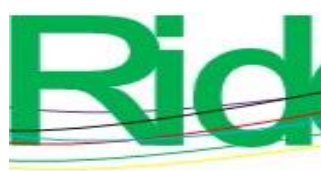

Revista Iberoamericana para la Investigación y el Desarrollo Educativo ISSN 2007 - 7467

Tabla 3. Frecuencia de inconvenientes y obstáculos fijos y no fijos en el radio de $800 \mathrm{~m}$ del Parque Central

\begin{tabular}{|c|c|c|c|c|c|c|c|c|c|}
\hline \multicolumn{2}{|c|}{ Obstáculos o dificultades fijos } & \multicolumn{6}{|c|}{ Ruta } & \multirow{3}{*}{$\begin{array}{c}\text { Total } \\
29\end{array}$} & \multirow{3}{*}{$\begin{array}{c}\text { NI } \\
\\
1\end{array}$} \\
\hline & & \multirow{2}{*}{$\begin{array}{l}1 \\
2\end{array}$} & \multirow{2}{*}{$\begin{array}{l}2 \\
2\end{array}$} & \multirow{2}{*}{$\begin{array}{c}3 \\
12\end{array}$} & \multirow{2}{*}{$\begin{array}{l}4 \\
2\end{array}$} & \multirow{2}{*}{$\begin{array}{l}5 \\
8\end{array}$} & \multirow{2}{*}{$\begin{array}{l}6 \\
3\end{array}$} & & \\
\hline Acera & Faltante & & & & & & & & \\
\hline & En mal estado & 3 & 0 & 5 & 3 & 4 & 1 & 16 & 2 \\
\hline & Bloqueada por elemento fijo & 1 & 1 & 2 & 1 & 5 & 3 & 13 & 4 \\
\hline & Demasiado estrecha & 1 & 1 & 0 & 1 & 1 & 1 & 5 & 9 \\
\hline \multirow[t]{3}{*}{ Rampa } & Faltante & 0 & 1 & 2 & 1 & 2 & 1 & 7 & 8 \\
\hline & En mal estado & 1 & 2 & 1 & 1 & 1 & 1 & 7 & 8 \\
\hline & $\begin{array}{l}\text { Rampa bloqueada por } \\
\text { elemento fijo }\end{array}$ & 0 & 0 & 0 & 0 & 0 & 0 & 0 & 19 \\
\hline \multirow[t]{3}{*}{ Pavimento } & Faltante & 0 & 1 & 2 & 0 & 1 & 0 & 4 & 10 \\
\hline & En mal estado & 0 & 2 & 1 & 0 & 0 & 0 & 3 & 12 \\
\hline & Suelto o baches & 0 & 0 & 0 & 8 & 2 & 5 & 15 & 3 \\
\hline \multirow[t]{3}{*}{ Horadación } & Hoyo en acera & 1 & 3 & 4 & 0 & 0 & 0 & 8 & 7 \\
\hline & Hoyo en acera & 2 & 1 & 1 & 1 & 0 & 5 & 10 & 6 \\
\hline & Zanja & 0 & 0 & 0 & 0 & 0 & 1 & 1 & 16 \\
\hline \multirow{9}{*}{$\begin{array}{l}\text { Discontinuidad } \\
\text { en el trayecto }\end{array}$} & Puesto de seguridad privada & 0 & 1 & 0 & 0 & 0 & 0 & 1 & 16 \\
\hline & Calle cerrada por seguridad & 1 & 2 & 0 & 0 & 0 & 0 & 3 & 12 \\
\hline & $\begin{array}{l}\text { Calle cerrada (otro motivo } \\
\text { fijo) }\end{array}$ & 0 & 0 & 0 & 0 & 0 & 0 & 0 & 19 \\
\hline & Rejas o cerco salvable & 0 & 0 & 0 & 0 & 2 & 0 & 2 & 15 \\
\hline & Tope reductor de velocidad & 2 & 9 & 2 & 0 & 0 & 0 & 13 & 4 \\
\hline & Dren, acequia o arroyo & 0 & 1 & 0 & 0 & 0 & 0 & 1 & 16 \\
\hline & Puente peatonal & 0 & 2 & 0 & 1 & 0 & 0 & 3 & 12 \\
\hline & Avenida principal & 1 & 1 & 1 & 0 & 0 & 1 & 4 & 10 \\
\hline & Total & 15 & 30 & 33 & 19 & 26 & 22 & 145 & \\
\hline \multicolumn{2}{|c|}{ Obstáculos o dificultades no fijos } & & & & & & & & \\
\hline
\end{tabular}




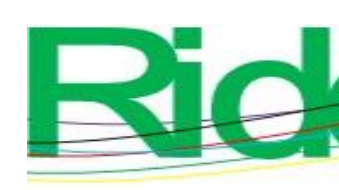

Revista Iberoamericana para la Investigación y el Desarrollo Educativo ISSN 2007 - 7467

\begin{tabular}{|c|c|c|c|c|c|c|c|c|c|}
\hline & $\begin{array}{l}\text { Acera bloqueada por } \\
\text { automóvil }\end{array}$ & 0 & 2 & 0 & 2 & 0 & 0 & 4 & 2 \\
\hline & $\begin{array}{l}\text { Acera bloqueada por material } \\
\text { de construcción }\end{array}$ & 1 & 1 & 0 & 0 & 0 & 0 & 2 & 4 \\
\hline & $\begin{array}{l}\text { Acera bloqueada por } \\
\text { anuncios }\end{array}$ & 0 & 0 & 0 & 1 & 0 & 1 & 2 & 4 \\
\hline & Desechos orgánicos & 1 & 0 & 0 & 0 & 0 & 0 & 1 & 6 \\
\hline & $\begin{array}{l}\text { Mercancía o muebles en la } \\
\text { acera }\end{array}$ & 0 & 0 & 0 & 1 & 0 & 0 & 1 & 6 \\
\hline & $\begin{array}{l}\text { Puestos de comida movibles } \\
\text { en acera }\end{array}$ & 0 & 0 & 2 & 0 & 1 & 0 & 3 & 3 \\
\hline & $\begin{array}{l}\text { Otros bloqueos de acera } \\
\text { (contenedores de basura, } \\
\text { macetas) }\end{array}$ & 3 & 12 & 4 & 12 & 6 & 5 & 42 & 1 \\
\hline & $\begin{array}{l}\text { Automóviles bloqueando } \\
\text { calle }\end{array}$ & 0 & 0 & 0 & 0 & 0 & 0 & 0 & 7 \\
\hline & $\begin{array}{lll}\text { Mercados } & \text { o vehículos } \\
\text { comerciales } & \end{array}$ & 0 & 0 & 0 & 0 & 0 & 0 & 0 & 7 \\
\hline & $\begin{array}{l}\text { Vehículos estacionados en } \\
\text { doble fila }\end{array}$ & 0 & 0 & 0 & 0 & 0 & 0 & 0 & 7 \\
\hline & $\begin{array}{l}\text { Vehículos estacionados en } \\
\text { batería }\end{array}$ & 0 & 0 & 0 & 0 & 0 & 0 & 0 & 7 \\
\hline & $\begin{array}{l}\text { Talleres mecánicos } \mathrm{o} \\
\text { vehículos en reparación }\end{array}$ & 0 & 0 & 0 & 0 & 0 & 0 & 0 & 7 \\
\hline & Vehículos abandonados & 0 & 0 & 0 & 0 & 0 & 0 & 0 & 7 \\
\hline & Cajas de tráiler & 0 & 0 & 0 & 0 & 0 & 0 & 0 & 7 \\
\hline & Postes o árboles tirados & 0 & 0 & 0 & 0 & 0 & 0 & 0 & 7 \\
\hline & Total & 5 & 15 & 6 & 16 & 7 & 6 & 55 & \\
\hline \multicolumn{10}{|c|}{$\begin{array}{l}\text { Nota: solo se muestran los obstáculos encontrados en alguna ruta. } \\
\text { NI = Nivel de importancia }\end{array}$} \\
\hline
\end{tabular}

Fuente: Elaboración propia 

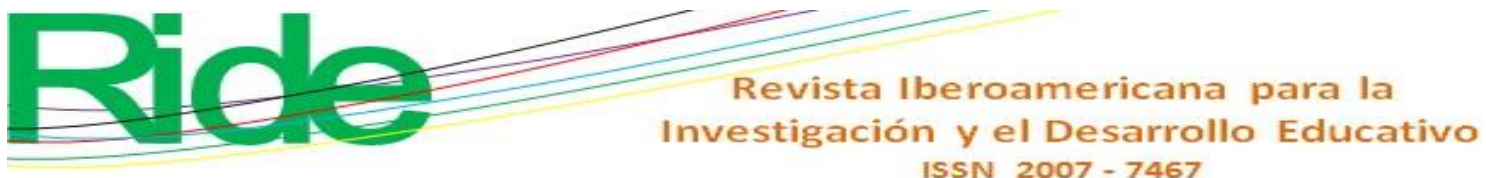

ISSN 2007 - 7467

Figura 3. Rutas uno, dos y tres y ejemplos de algunos obstáculos

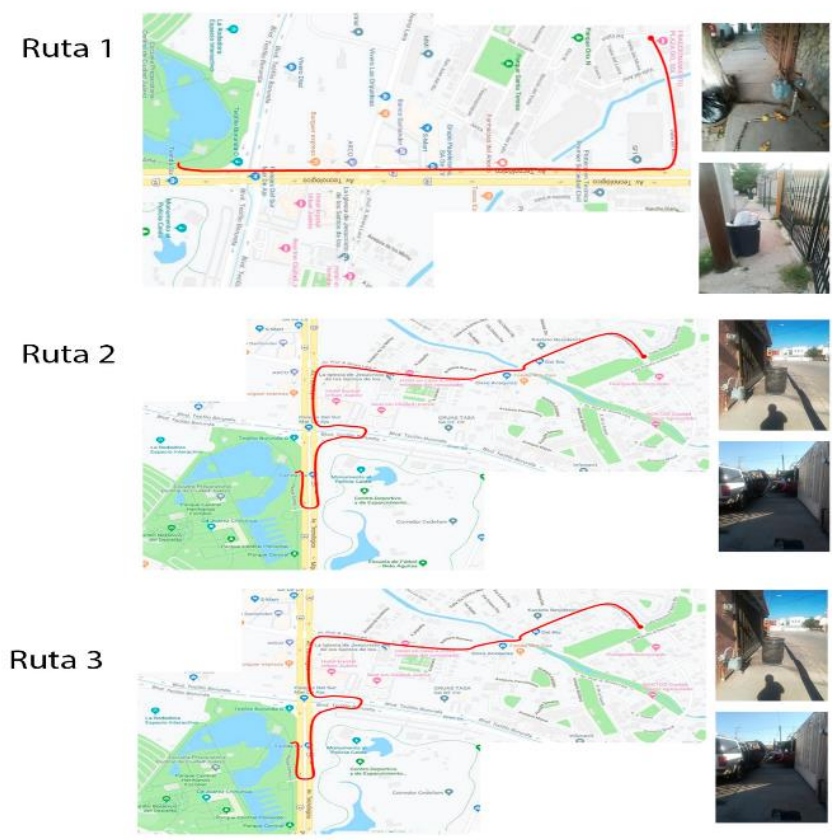

Fuente: Elaboración propia

Figura 4. Rutas cuatro, cinco y seis y ejemplos de algunos obstáculos

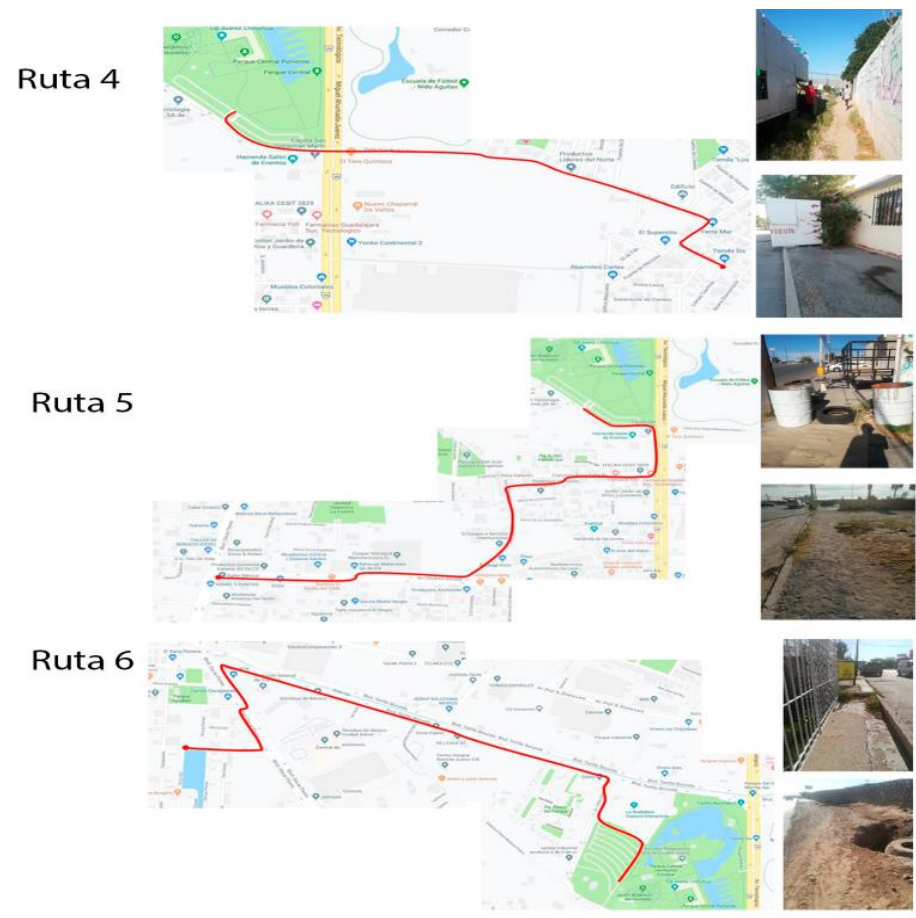

Fuente: Elaboración propia 


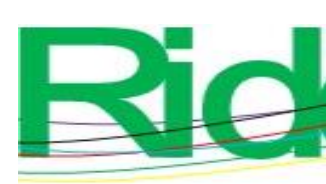

Revista Iberoamericana para la Investigación y el Desarrollo Educativo ISSN 2007- 7467

Figura 5. Frecuencia de obstáculos fijos en las rutas

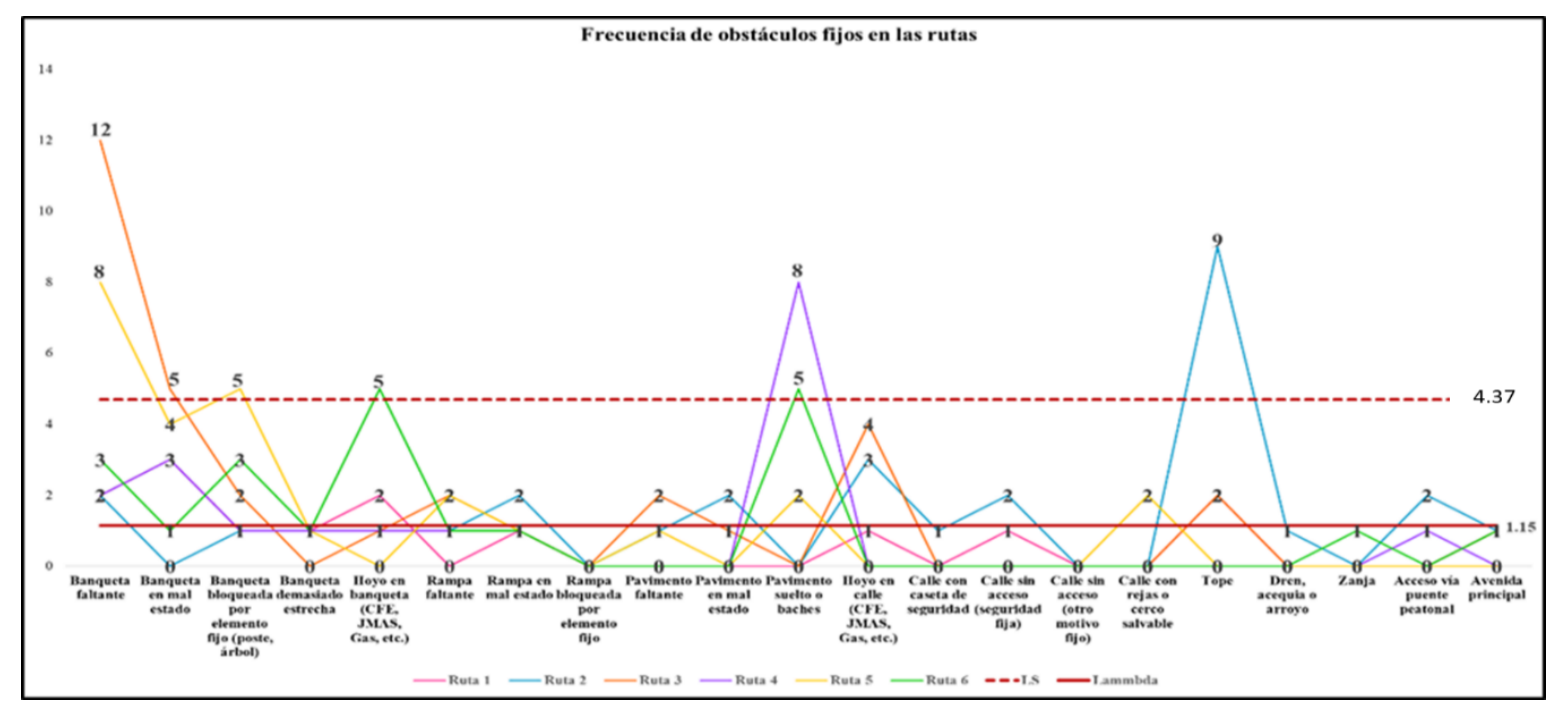

Fuente: Elaboración propia

El valor del límite superior de $\mathrm{S}_{\mathrm{c}}$ se ubica en 4.37 obstáculos. Resaltan las frecuencias de aquellos obstáculos que rebasan este límite y que son atribuibles precisamente a la ruta donde se presentaron. Así, en la ruta uno, se observan frecuencias menores a la frontera con la variación aleatoria, lo que sugiere que no está presente un obstáculo fijo dominante en esta ruta. Por su parte, en la ruta dos el obstáculo fijo "Tope reductor de velocidad" es específicamente atribuible a esta ruta, más allá de la variación aleatoria. Lo mismo pasa con los obstáculos “acera faltante" y "acera en mal estado", que son específicos para la ruta tres. Mientras que el obstáculo fijo "pavimento suelto o baches" destaca en la ruta cuatro. Los obstáculos “acera faltante" y "acera bloqueada" son atribuibles a la ruta cinco. Por último, a la ruta seis le son atribuibles los obstáculos fijos "hoyo en acera" y "pavimento suelto o baches".

La frecuencia con la que se presentan los obstáculos fijos es útil para identificar aquellos que son más comunes en cada trayecto estudiado. Sin embargo, no todos los obstáculos fijos tienen la misma importancia: algunos son insalvables, otros son inevitables pero salvables y otros se pueden evitar durante el trayecto. Se penalizaron los obstáculos fijos con base en un factor de ponderación $(w=5,3,1)$, donde 5 es muy crítico, 3 es importante y 1 es no crítico y menos importante. Los resultados se muestran en la tabla 4 y tabla 5; la representación gráfica en la figura 6. 
Tabla 4. Obstáculos fijos atribuibles a las rutas consideradas en el radio de $800 \mathrm{~m}$ del

Parque Central

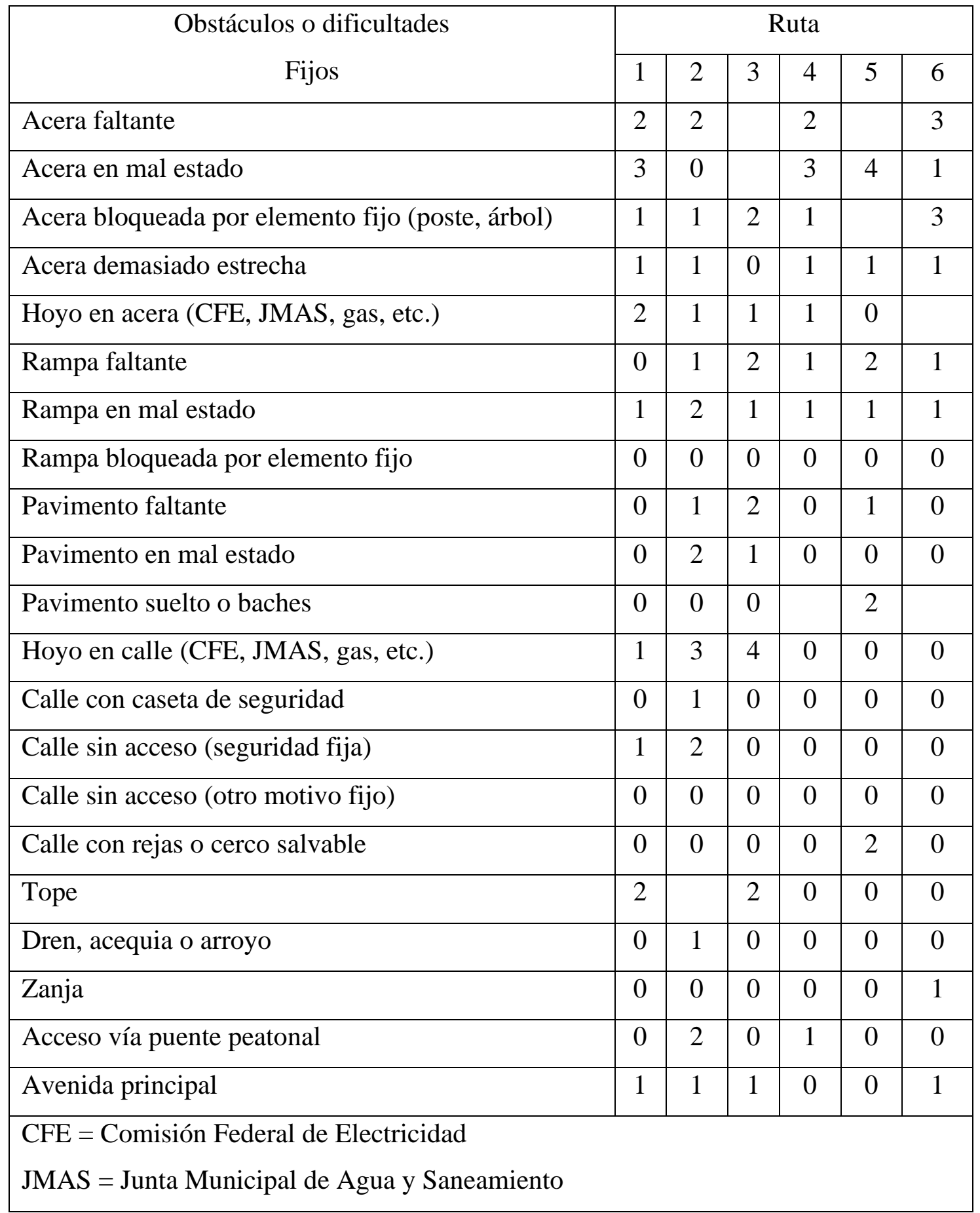

Fuente: Elaboración propia 


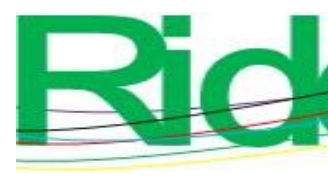

Revista Iberoamericana para la Investigación y el Desarrollo Educativo ISSN 2007 - 7467

Tabla 5. Penalización de obstáculos fijos en las rutas consideradas

\begin{tabular}{|c|c|c|c|c|c|c|c|c|c|}
\hline \multirow{2}{*}{$\begin{array}{c}\text { Obstáculos o dificultades } \\
\text { Fijos }\end{array}$} & \multirow[b]{2}{*}{$W$} & \multicolumn{6}{|c|}{ Ruta } & \multirow[t]{2}{*}{ Total } & \multirow[t]{2}{*}{ NI } \\
\hline & & 1 & 2 & 3 & 4 & 5 & 6 & & \\
\hline Acera faltante & 5 & 10 & 10 & 60 & 10 & 40 & 15 & 145 & 1 \\
\hline Acera en mal estado & 3 & 9 & 0 & 15 & 9 & 12 & 3 & 48 & 2 \\
\hline Acera bloqueada por elemento fijo & 3 & 3 & 3 & 6 & 3 & 15 & 9 & 39 & 4 \\
\hline Acera demasiado estrecha & 3 & 3 & 3 & 0 & 3 & 3 & 3 & 15 & 10 \\
\hline $\begin{array}{l}\text { Hoyo en acera (CFE, JMAS, gas, } \\
\text { etc.) }\end{array}$ & 3 & 6 & 3 & 3 & 3 & 0 & 15 & 30 & 7 \\
\hline Rampa faltante & 5 & 0 & 5 & 10 & 5 & 10 & 5 & 35 & 6 \\
\hline Rampa en mal estado & 3 & 3 & 6 & 3 & 3 & 3 & 3 & 21 & 8 \\
\hline Rampa bloqueada por elemento fijo & 3 & 0 & 0 & 0 & 0 & 0 & 0 & 0 & 20 \\
\hline Pavimento faltante & 5 & 0 & 5 & 10 & 0 & 5 & 0 & 20 & 9 \\
\hline Pavimento en mal estado & 3 & 0 & 6 & 3 & 0 & 0 & 0 & 9 & 13 \\
\hline Pavimento suelto o baches & 3 & 0 & 0 & 0 & 24 & 6 & 15 & 45 & 3 \\
\hline $\begin{array}{l}\text { Hoyo en calle (CFE, JMAS, gas, } \\
\text { etc.) }\end{array}$ & 1 & 1 & 3 & 4 & 0 & 0 & 0 & 8 & 15 \\
\hline Calle con caseta de seguridad & 1 & 0 & 1 & 0 & 0 & 0 & 0 & 1 & 19 \\
\hline Calle sin acceso (seguridad fija) & 5 & 5 & 10 & 0 & 0 & 0 & 0 & 15 & 10 \\
\hline Calle sin acceso (otro motivo fijo) & 5 & 0 & 0 & 0 & 0 & 0 & 0 & 0 & 20 \\
\hline Calle con rejas o cerco salvable & 1 & 0 & 1 & 0 & 0 & 2 & 0 & 3 & 16 \\
\hline Tope & 3 & 6 & 27 & 6 & 0 & 0 & 0 & 39 & 4 \\
\hline Dren, acequia o arroyo & 3 & 0 & 3 & 0 & 0 & 0 & 0 & 3 & 16 \\
\hline Zanja & 3 & 0 & 0 & 0 & 0 & 0 & 3 & 3 & 16 \\
\hline Acceso vía puente peatonal & 3 & 0 & 6 & 0 & 3 & 0 & 0 & 9 & 13 \\
\hline \multirow[t]{2}{*}{ Avenida principal } & 3 & 3 & 3 & 3 & 0 & 0 & 3 & 12 & 12 \\
\hline & Total & 49 & 95 & 123 & 63 & 96 & 74 & 500 & \\
\hline & & & & & & & \multicolumn{2}{|c|}{$w=$ Factor de ponderación } & \\
\hline
\end{tabular}

Fuente: Elaboración propia 


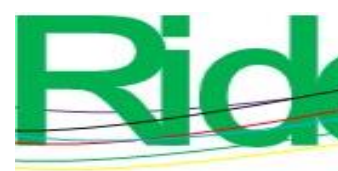

Revista Iberoamericana para la Investigación y el Desarrollo Educativo ISSN 2007 - 7467

empatadas en cuarto lugar estas dos últimas. Los demás tipos de obstáculos son menos relevantes para este análisis.

Los obstáculos móviles durante el trayecto son coyunturales. La mayoría se presenta por errores, omisiones, incumplimientos de la normatividad local o simplemente malos hábitos ciudadanos. No obstante, para efectos de acceder al parque, son factores que inciden negativamente. Por mucho, más de 10 veces en relación con el segundo lugar, la categoría "otros bloqueos de acera" (que incluye elementos móviles, tales como macetas no fijas y contenedores de basura, principalmente) se posiciona en primer lugar. Muy de lejos le siguen “acera bloqueada por automóvil” y "puesto móvil de comida en acera”.

Una vez identificados los principales obstáculos y su criticidad, es pertinente recordar que los gobiernos locales usualmente tienen diagnosticados los problemas de su ámbito de competencia. La constante que impide su solución radica en las restricciones presupuestales, sobre todo en materia de espacios verdes urbanos y sus entornos (Aalbers, Kamphorst y Langers, 2019; Jansson, Vogel, Fors y Randrup, 2019; Sainz y Martinez, 2021). En este caso, la autoridad local de Ciudad Juárez tiene más a la mano la intervención en los obstáculos fijos y parece tener al menos tres alternativas a la vista para mejorar la accesibilidad del parque:

a) La primera sería mejorar la accesibilidad radial en lo general, lo que de suyo rebasaría la capacidad presupuestaria del municipio, ya que la ciudad sufre de problemas sistémicos de degradación del entorno construido y malas prácticas ciudadanas que tomaría tiempo revertir (IMIP, 2021; Sánchez, 20 de marzo de 2019).

b) La segunda alternativa sería llevar todos los obstáculos con frecuencias superiores a cuatro precisamente a este nivel y llegar a una condición sistémica de peatonabilidad para todas las rutas; sin embargo, este rumbo de acción parece carecer de sentido común, ya que sería un despropósito, por ejemplo, reparar un bache (de los cinco que se encontraron) simplemente para cumplir con esta condición.

c) La tercera alternativa sería reparar o subsanar las cinco categorías de obstáculos que resultaron más críticos; esta alternativa sería focalizada y no requeriría de una partida presupuestal mayor o extraordinaria.

La tabla 6 resume los escenarios disponibles para la autoridad municipal y la forma en la que cambiaría el análisis estadístico si se llevaran a cabo. 


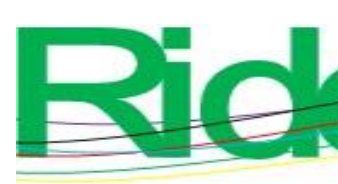

Revista Iberoamericana para la
Investigación y el Desarrollo Educativo
ISSN $2007-7467$

evaluación económica de las alternativas presentadas está más allá del propósito de este artículo.

\section{Discusión}

El análisis estadístico de Poisson, considerando que la ciudad es un sistema y que el entorno construido, que en gran medida define la peatonabilidad, puede ser estudiado como un proceso en el que es factible separar las condiciones inherentes a la dinámica de la ciudad misma (variación aleatoria) de las causas atribuibles a determinadas rutas (variación especial o atribuible), reveló estrategias eficaces de gestión para el Gobierno municipal.

La mejora de la calidad de la accesibilidad del Parque Central se manifiesta al combinar el análisis estadístico y el de criticidad, lo que permite enfocarse en los factores relevantes, en este caso los obstáculos que más negativamente afectan la peatonabilidad. El radio de 800 m que se consideró para el análisis no es un entramado de una diversidad de rutas, ya que el parque se localiza, por un lado, en la intersección de dos avenidas principales de la ciudad; y por otro, las rutas que se alejan de las trayectorias lógicas de acceso al parque no definen su accesibilidad radial. Esto es, las rutas consideradas en este estudio, o segmentos de estas, serían las que ulteriormente la gran mayoría de las personas utilizaría para caminar al parque dentro del radio de estudio.

De la gran diversidad de obstáculos que limitan la peatonabilidad y afectan la calidad de la accesibilidad radial, tres se refieren a las aceras, un elemento esencial, no solo para la peatonabilidad, sino para la sustentabilidad urbana en su conjunto y hasta para la democracia misma, como algunas investigaciones lo han destacado (Gunn, Lee, Geelhoed, Shiell y Giles, 2014; Landin, 23 de febrero de 2016; Osama y Sayed, 2017; Vallejo, Cantillo y Rodriguez, 2020). Entonces, el enfoque adoptado en este trabajo reitera la importancia de las aceras, sobre todo para los grupos vulnerables de la población, además de brindar rumbo a la gestión gubernamental hacia su mejoramiento (Duan, Wagner, Zhang, Wulff y Brehm, 2018; Guo et al., 2019).

En el mismo tema, el mal estado actual de las aceras y los obstáculos no fijos presentes obligan a las personas a caminar por las calles. En este sentido, los dos restantes obstáculos críticos que también se revelaron del análisis son abordables sin que signifiquen un gasto excesivo para la autoridad. Así, el pavimento suelto y los baches son factibles de ser 


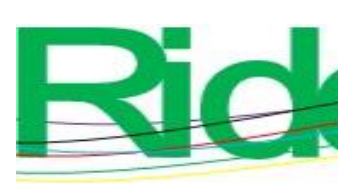

Revista Iberoamericana para la Investigación y el Desarrollo Educativo ISSN 2007 - 7467

reparados como parte del programa rutinario de bacheo de la ciudad. Por otra parte, los topes reductores de velocidad son la causa de fallas mecánicas y de congestionamiento de automotores en las calles; es frecuente que los topes reductores sean colocados por los vecinos sin el cumplimiento de la ordenanza municipal (Juárez, 2021). Estos obstáculos pueden ser removidos y en su lugar colocar señalética y vigilancia vial temporal en lo que los automovilistas se apegan a la normatividad y a las nuevas condiciones.

En lo que respecta a los obstáculos no fijos, se trata de un problema recurrente en la ciudad (Sosa, 2015). En este sentido, la educación cívica es un factor fundamental para ir convenciendo a los ciudadanos de respetar las aceras y las calles, de forma que se permita el libre tránsito de personas caminando. Al respecto, el Gobierno municipal mantiene una campaña agresiva para hacer buen uso de las aceras (Sánchez, 20 de marzo de 2019; El Diario de Juárez, 13 de abril de 2019), solo cabe esperar que estos esfuerzos continúen y que cada vez haya más conciencia ciudadana acerca de la importancia y los beneficios de caminar la ciudad con aceras libres de obstáculos.

En términos generales, este enfoque tiene como fortaleza el que el análisis es simple y los resultados son fácilmente aplicables en áreas donde el entorno construido puede ser identificado y registrado dentro de un radio caminable. Otra ventaja es que proporciona un perfil de criticidad que permite reconocer obstáculos con mayor trascendencia y enfocar los esfuerzos de gestión municipal en los aspectos más relevantes que inciden en la peatonabilidad. Por otra parte, quizás sea menos pertinente para el estudio de polígonos urbanos completos o rutas muy extensas, fuera del rango caminable de $800 \mathrm{~m}$. Para tales enfoques, quizás sea más adecuado el abordaje con SIG, bases de datos oficiales o aplicaciones de teléfonos inteligentes (Telega et al., 2021; Yun, Zegras y Palencia, 2019).

Los resultados encontrados permitieron identificar que los recursos desarrollados son insuficientes o la gestión inadecuada, el estado de conservación del entorno construido se ve disminuido, la infraestructura urbana se daña y la población se ve perjudicada por esta degradación; este grado de desorden está directamente asociado con la disminución de la sustentabilidad urbana (Fistola, Gargiulo y La Rocca, 2020).

En la temática de exclusividad y accesibilidad del parque se encontró que las características cumplen con las necesidades de que el parque urbano sea incluyente y accesible como un territorio de encuentro y de interacción que fomenta la diversidad, la cohesión social y promueve la salud física y mental, al tiempo que fortalece la sustentabilidad 


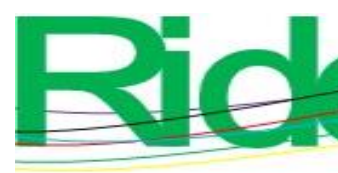

Revista Iberoamericana para la Investigación y el Desarrollo Educativo ISSN 2007 - 7467

de la ciudad (Grilli, Mohan y Curtis, 2020; Shashank y Schuurman, 2019). Los resultados revelan que la dinámica del proceso urbano en materia de peatonabilidad es lenta. Y en este sentido, la literatura incluye un conjunto de estudios de caso con temporalidad transversal y con diferentes técnicas en los cuales la importancia de la peatonabilidad se pone de manifiesto (Humberto et al., 2019; Maghelal y Capp, 2011; Shashank y Schuurman, 2019).

\section{Conclusiones}

En el contexto de su naturaleza sistémica y entrópica, la ciudad procesa recursos y energía, además de requerir acciones de gestión para mantener su funcionalidad y frenar su deterioro. Se concluye que el estudio revela la existencia de recursos insuficientes debido a la gestión inadecuada. Derivado de lo anterior, el estado de conservación del entorno construido se ve disminuido, la infraestructura urbana se daña y la población se ve perjudicada por esta degradación.

Entonces, se concluye que la adecuada gestión del entorno construido es fundamental para permitir el traslado de personas a pie (peatonabilidad) dentro del radio de influencia del parque, por lo que este abordaje urbano definió que la calidad de la accesibilidad radial de un espacio público es débil si no existe una gestión que la considere una prioridad. Además, se evidenciaron las condiciones existentes en el momento del estudio, lo que pone de manifiesto la importancia de esta investigación y sus aportaciones inmediatas al problema existente.

La dinámica del proceso urbano en materia de peatonabilidad encontrada reveló que es lenta. Consecuentemente, el estudio concluye que la problemática prevalente abona en su gestación a las escasas investigaciones que consideran la condición sistémica del entorno construido en el área de influencia de un parque urbano y, por lo tanto, se contribuye a la disponibilidad de elementos de discusión de las afectaciones directas sobre los accesos a los parques urbanos de gran escala. Lo anterior lleva a concluir que este trabajo y el abordaje de la visión sistémica e incorporación de la perspectiva innovadora de análisis, no solo puso de manifiesto los factores críticos que afectan la calidad de la accesibilidad radial del Parque Central, sino que adicionalmente reveló líneas de acción de gestión eficaces para el gobierno municipal. Por lo anterior, se concluye que el enfoque de CEP, no obstante ser una técnica de monitoreo longitudinal, es pertinente para identificar el estado de la peatonabilidad y 

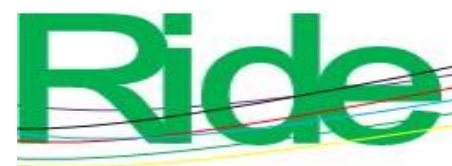

Revista Iberoamericana para la Investigación y el Desarrollo Educativo

ISSN 2007 - 7467

evaluar la calidad de la accesibilidad radial hacia un espacio público en un ejercicio de temporalidad transversal, por lo que su uso es recomendable.

\section{Contribuciones a futuras líneas de investigación}

Para finalizar, se recomienda estudiar el uso de aplicaciones inteligentes, georreferenciadas en tiempo real, o al menos con un corto tiempo de retraso (Guo et al., 2019; Moura et al., 2017; Telega et al., 2021). Lo anterior permitiría a un peatón visualizar desde su dispositivo móvil la ruta más accesible para llegar al parque, o bien anticipar eventualidades en el trayecto. Este estudio puede complementarse con una investigación longitudinal que muestre el progreso o degradación del proceso urbano en relación con el entorno construido, lo que por ahora constituye un gran hueco en la literatura, al menos en Latinoamérica. Se recomienda también estudiar los eventuales modelos de gobernanza para mejorar la peatonabilidad, la calidad de la accesibilidad radial y la calidad del espacio verde urbano en lo general (Sainz y Martinez, 2021). La participación de la ciudadanía, en confluencia con la autoridad municipal, es quizás la mejor estrategia de gestión de largo plazo para incrementar la peatonabilidad del área de influencia del Parque Central y mejorar la calidad de la accesibilidad radial, reforzando los servicios medioambientales, sociales y económicos que brinda el parque. 


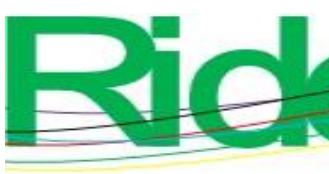

Revista Iberoamericana para la
Investigación y el Desarrollo Educativo
ISSN $2007-7467$

\section{Referencias}

Aalbers, C. B. E. M., Kamphorst, D. A. and Langers, F. (2019). Fourteen local governance initiatives in greenspace in urban areas in the Netherlands. Discourses, success and failure factors, and the perspectives of local authorities. Urban Forestry \& Urban Greening, 42, 82-99. Retrieved from https://doi.org/10.1016/j.ufug.2019.04.019.

Asadi-Shekari , Z., Moeinaddini, M. and Zaly, M. (2013). Non-motorised Level of Service: Addressing Challenges in Pedestrian and Bicycle Level of Service. Transport Reviews, 33(2), 166-194. Retrieved from https://doi.org/10.1080/01441647.2013.775613.

Cambra, P. (2012). Pedestrian Accessibility and Attractiveness Indicators for Walkability Assessment. Retrieved from https://www.semanticscholar.org/paper/PedestrianAccessibility-and-Attractiveness-for-Cambra-

Ordenamento/ee089f66f347e662905d92ef1bbe16d7d74a9aa1?p2df.

Centro Estatal de Autonomía Personal y Ayudas Físicas [Ceapat]. (1996). Concepto europeo de accesibilidad. Recuperado de http://www.ceapat.es/InterPresent2/groups/imserso/documents/binario/concepto_eur opeo_de_accesibili.pdf.

Cubukcu, E. (2013). Walking for Sustainable Living. Procedia - Social and Behavioral Sciences, 85, 33-42. Retrieved from https://doi.org/10.1016/J.SBSPRO.2013.08.335.

El Diario de Juárez. (13 de abril de 2019). Multan a más de 10 mil 200 automovilistas por estacionarse en banquetas. El Diario.mx. Recuperado de https://diario.mx/juarez/multan-a-mas-de-10-mil-200-automovilistas-porestacionarse-en-banquetas-20190413-1502207.

Duan, Y., Wagner, P., Zhang, R., Wulff, H. and Brehm, W. (2018). Physical activity areas in urban parks and their use by the elderly from two cities in China and Germany. Landscape and Urban Planning, 178, 261-269. Retrieved from https://doi.org/10.1016/J.LANDURBPLAN.2018.06.009.

Duncan, D. T., Aldstadt, J., Whalen, J., Melly, S. J. and Gortmaker, S. L. (2011). Validation of Walk Score® for Estimating Neighborhood Walkability: An Analysis of Four US Metropolitan Areas. International Journal of Environmental Research and Public Health, 8(11), 4160-4179. Retrieved from https://doi.org/10.3390/ijerph8114160. 


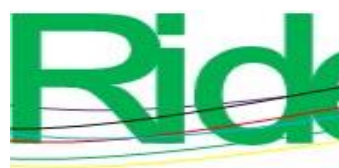

Revista Iberoamericana para la Investigación y el Desarrollo Educativo ISSN $2007-7467$

Fistola, R., Gargiulo, C. and La Rocca, R. A. (2020). Rethinking vulnerability in citysystems: A methodological proposal to assess "urban entropy." Environmental Impact Assessment Review, 85. Retrieved from https://doi.org/10.1016/j.eiar.2020.106464.

Florindo, A. A., dos Anjos, J. P., Vizeu, L., Roque, D., Souza, B., Antunes, M., Gunn, L., Mavoa, S., Turrell, G. and Goldbaum, M. (2019). Walking for transportation and built environment in Sao Paulo city, Brazil. Journal of Transport \& Health, 15. Retrieved from https://doi.org/10.1016/J.JTH.2019.100611.

Forsyth, A. (2015). What is a walkable place? The walkability debate in urban design. Urban Design International, 20(4), 274-292. Retrieved from https://doi.org/10.1057/udi.2015.22.

Grilli, G., Mohan, G. and Curtis, J. (2020). Public park attributes, park visits, and associated health status. Landscape and Urban Planning, 199. Retrieved from https://doi.org/10.1016/j.landurbplan.2020.103814.

Gunn, L. D., Lee, Y., Geelhoed, E., Shiell, A. and Giles, B. (2014). The cost-effectiveness of installing sidewalks to increase levels of transport-walking and health. Preventive Medicine, 67, 322-329. $\quad$ Retrieved from https://doi.org/10.1016/J.YPMED.2014.07.041.

Guo, S., Song, C., Pei, T., Liu, Y., Ma, T., Du, Y., Chen, J., Fan, Z., Tang, X., Peng, Y. and Wang, Y. (2019). Accessibility to urban parks for elderly residents: Perspectives from mobile phone data. Landscape and Urban Planning, 191, 103642. Retrieved from https://doi.org/10.1016/J.LANDURBPLAN.2019.103642.

Humberto, M., Laboissière, R., Giannotti, M., Marte, C. L., Cruz, D. A. and Primon, H. (2019). Walking and walkability: do built environment measures correspond with pedestrian activity? Ambiente Construído, 19(4), 23-36. Retrieved from https://doi.org/10.1590/s1678-86212019000400341.

Hussein, N. (2018). The Pedestrianisation and Its Relation with Enhancing Walkability in Urban Spaces. Journal of Contemporary Urban Affairs, 2(1), 102-112. Retrieved from https://doi.org/10.25034/ijcua.2018.3666.

Instituto Municipal de Investigación y Planeación [IMIP]. (2020). Vialidades con y sin pavimento en el municipio de Juárez. Recuperado de https://www.imip.org.mx/imip/node/78, 


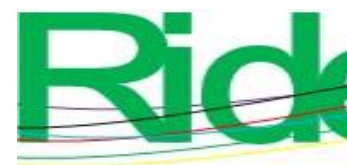

Revista Iberoamericana para la
Investigación y el Desarrollo Educativo
ISSN $2007-7467$

Instituto Municipal de Investigación y Planeación [IMIP]. (2021). Radiografía socioeconómica del municipio de Juárez 2020, así comenzó 2021. Ciudad Juárez, México: Instituto Municipal de Investigación y Planeación. Recuperado de https://www.imip.org.mx/imip/files/radiografia/Radiografia2020-2021.pdf.

Jansson, M., Vogel, N., Fors, H. and Randrup, T. B. (2019). The governance of landscape management: new approaches to urban open space development. Landscape $\begin{array}{llll}\text { Research, } & \text { 44(8), } & \text { Retrieved }\end{array}$ https://doi.org/10.1080/01426397.2018.1536199.

Juárez, C. (3 de abril de 2021). Ilegal que ciudadanos pongan topes en calles. Netnoticias.mx. Recuperado de https://netnoticias.mx/juarez/ilegal-que-ciudadanos-pongan-topesen-calles/.

Kennedy, C., Pincetl, S. and Bunje, P. (2011). The study of urban metabolism and its applications to urban planning and design. Environmental Pollution, 159(8-9), 19651973. Retrieved from https://doi.org/10.1016/j.envpol.2010.10.022.

Landin, J. M. (23 de febrero de 2016). Las banquetas de México. Nexos. Recuperado de https://labrujula.nexos.com.mx/?p=698.

Maghelal, P. K. and Capp, C. J. (2011). Walkability: a review of existing pedestrian indices. URISA Journal, 23(2), 5-20. Retrieved from https://go.gale.com/ps/i.do?p=AONE\&sw=w\&issn=10458077\&v=2.1\&it=r\&id=G ALE\%7CA283455689\&sid=googleScholar\&linkaccess=fulltext.

Moura, F., Cambra, P. and Gonçalves, A. B. (2017). Measuring walkability for distinct pedestrian groups with a participatory assessment method: A case study in Lisbon. Landscape and Urban Planning, 157, 282-296. Retrieved from https://doi.org/10.1016/j.landurbplan.2016.07.002.

Osama, A. and Sayed, T. (2017). Evaluating the impact of connectivity, continuity, and topography of sidewalk network on pedestrian safety. Accident Analysis \& Prevention, 107, 117-125. Retrieved from https://doi.org/10.1016/J.AAP.2017.08.001.

Pelorosso, R., Gobattoni, F. and Leone, A. (2017). The low-entropy city: A thermodynamic approach to reconnect urban systems with nature. Landscape and Urban Planning, 168, 22-30. Retrieved from https://doi.org/10.1016/J.LANDURBPLAN.2017.10.002. 


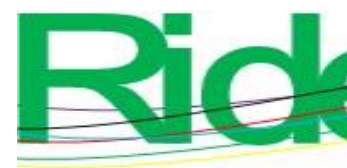

Revista Iberoamericana para la
Investigación y el Desarrollo Educativo
ISSN $2007-7467$

Peñalosa, G. (2018). Creando ciudades exitosas y saludables para todos. Congreso Internacional de Parques Urbanos. https://www.youtube.com/watch?v=TrsAQFcNus

Pinson, G. and Morel, C. (2016). The Neoliberal City - Theory, Evidence, Debates. Territory, Politics, Governance, 4(2), 137-153. Retrieved from https://doi.org/10.1080/21622671.2016.1166982.

Rahaman, K. R., Lourenço, J. M. and Viegas, J. M. (2012). Perceptions of Pedestrians and Shopkeepers in European Medium-Sized Cities: Study of Guimarães, Portugal. Journal of Urban Planning and Development, 138(1), 26-34. Retrieved from https://doi.org/10.1061/(asce)up.1943-5444.0000094.

Sainz, J. and Martinez, A. L. (2021). Governance of Urban Green Spaces across Latin America - Insights from Semi-Structured Interviews to Managers Amid COVID-19. SSRN Electronic Journal. Retrieved from https://doi.org/10.2139/ssrn.3782285.

Sánchez, M. (20 de marzo de 2019). Banquetas no son para estacionarse: pide Cabada respetar reglamento. Puente Libre.mx. Recuperado de http://puentelibre.mx/noticia/banquetas_no_son_para_estacionarse_pide_cabada_res petar_reglamento/.

Secretaría de Desarrollo Urbano y Vivienda [Seduvi]. (2016). Manual de normas técnicas de accesibilidad. Ciudad de México, México: Secretaría de Desarrollo Urbano y Vivienda. Recuperado de http://www.data.seduvi.cdmx.gob.mx/portal/images/banners/banner_derecho/docum entos/Manual_Normas_Tecnicas_Accesibilidad_2016.pdf.

Serrano, M. F., Jaramillo, L. F., Campos, C. A. y Galindo, N. J. (2013). Instrumento para la evaluación de la accesibilidad con criterios de diseño universal. Revista Virtual Universidad Católica del Norte, (39), 143-151.

Shashank, A. and Schuurman, N. (2019). Unpacking walkability indices and their inherent assumptions. Health and Place, 55, 145-154. Retrieved from https://doi.org/10.1016/j.healthplace.2018.12.005.

Shewhart, W. A. (1986). Statistical Method from the Viewpoint of Quality Control. New York, United States: Dover Publications. 


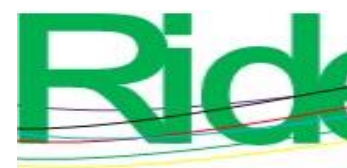

Revista Iberoamericana para la
Investigación y el Desarrollo Educativo
ISSN $2007-7467$

Sosa, L. del C. (2015). Padece la ciudad anarquía en el uso de banquetas - El Diario. Diario de Juárez. https://diario.mx/Local/2015-03-02_ed3c7bf6/padece-la-ciudad-anarquiaen-el-uso-de-banquetas/

Contreras, R. y Aguilar, O. C. (2012). Desarrollo sostenible (semblanza histórica). Revista del Centro de Investigación de la Universidad La Salle, 10(37), 101-121. Recuperado de http://revistasinvestigacion.lasalle.mx/index.php/recein/article/view/102/293.

Subirats, J. (2016). Explorar el espacio público como bien común. Debates conceptuales y de gobierno en la ciudad fragmentada. En Ramírez, P. (coord. ${ }^{a}$ ), La reinvención del espacio público en la ciudad fragmentada (pp. 99-134). Ciudad de México, México: Universidad Autónoma de México, Instituto de Investigaciones Sociales.

Tal, G. and Handy, S. (2012). Measuring Nonmotorized Accessibility and Connectivity in a Robust Pedestrian Network. Transportation Research Record: Journal of the Transportation Research Board, 2299(1), 48-56. Retrieved from https://doi.org/10.3141/2299-06.

Talavera, R. and Soria, J. A. (2015). Q-PLOS, developing an alternative walking index. A method based on urban design quality. Cities, 45, 7-17. Retrieved from https://doi.org/10.1016/j.cities.2015.03.003.

Telega, A., Telega, I. and Bieda, A. (2021). Measuring Walkability with GIS-Methods Overview and New Approach Proposal. Sustainability, 13(4), 1883. Retrieved from https://doi.org/10.3390/SU13041883.

Vallejo, J. A., Cantillo, V. and Rodriguez, A. (2020). A perception-based cognitive map of the pedestrian perceived quality of service on urban sidewalks. Transportation Research Part F: Traffic Psychology and Behavior, 73, 107-118. Retrieved from https://doi.org/10.1016/J.TRF.2020.06.013.

von Bertalanffy, L. (1976). General System Theory (1 ${ }^{\text {st }}$ ed.). New York, United States: Geroge Braziller.

Walpole, R. E., Myers, R. H., Myers, S. L. y Ye, K. (2012). Probabilidad y estadística para ciencias e ingeniería (9. a ed.). Naucalpan de Juárez, México: Pearson Educación.

Wu, J. (2014). Urban ecology and sustainability: The state-of-the-science and future directions. Landscape and Urban Planning, 125, 209-221. Retrieved from https://doi.org/10.1016/j.landurbplan.2014.01.018. 

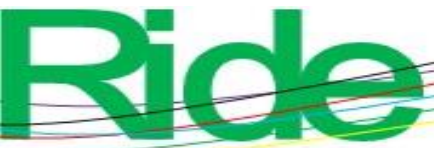

Yun, H. Y., Zegras, C. and Palencia, D. H. (2019). "Digitalizing Walkability": Comparing Smartphone-Based and Web-Based Approaches to Measuring Neighborhood Walkability in Singapore. Journal of Urban Technology, 26(3), 3-43. Retrieved from https://doi.org/10.1080/10630732.2019.1625016.

Zuniga, A. A., Orr, B. J., Gimblett, R. H., Chalfoun, N. V., Guertin, D. P. and Marsh, S. E. (2017). Neighborhood Design, Physical Activity, and Wellbeing: Applying the Walkability Model. International Journal of Environmental Research and Public Health, 14(1), 76. Retrieved from https://doi.org/10.3390/IJERPH14010076.

Zuniga, A. A., Stoker, P., Gimblett, R. H., Orr, B. J., Marsh, S. E., Guertin, D. P. and Chalfoun, N. V. (2019). Exploring the influence of neighborhood walkability on the frequency of use of greenspace. Landscape and Urban Planning, 190. Retrieved from https://doi.org/10.1016/J.LANDURBPLAN.2019.103609. 


\begin{tabular}{|c|c|}
\hline Rol de Contribución & Autor (es) \\
\hline Conceptualización & $\begin{array}{l}\text { Diego Adiel Sandoval Chávez (principal), Ana Córdova y } \\
\text { Vázquez (apoya), Aida Yarira Reyes Escalante (apoya). }\end{array}$ \\
\hline Metodología & $\begin{array}{l}\text { Diego Adiel Sandoval Chávez (principal), Aida Yarira Reyes } \\
\text { Escalante (apoya), Luz Elena Terrazas Mata (apoya). }\end{array}$ \\
\hline Software & $\begin{array}{l}\text { Diego Adiel Sandoval Chávez (principal), Aida Yarira Reyes } \\
\text { Escalante (igual), Luz Elena Tarango Hernández (apoya). }\end{array}$ \\
\hline Validación & $\begin{array}{l}\text { Aida Yarira Reyes Escalante (principal), Ana Córdova y } \\
\text { Vázquez (apoya), Diego Adiel Sandoval Chávez (apoya) }\end{array}$ \\
\hline Análisis Formal & $\begin{array}{l}\text { Diego Adiel Sandoval Chávez (principal), Aida Yarira Reyes } \\
\text { Escalante (apoya), Luz Elena Terrazas Mata (apoya). }\end{array}$ \\
\hline Investigación & $\begin{array}{l}\text { Diego Adiel Sandoval Chávez (principal), Aida Yarira Reyes } \\
\text { Escalante (igual), Ana Córdova y Vázquez (igual), Luz Elena } \\
\text { Tarango Hernández (apoya), Luz Elena Terrazas Mata (apoya). }\end{array}$ \\
\hline Recursos & $\begin{array}{l}\text { Aida Yarira Reyes Escalante (principal), Luz Elena Terrazas } \\
\text { Mata (apoya), Luz Elena Tarango Hernández (apoya) }\end{array}$ \\
\hline Curación de datos & $\begin{array}{l}\text { Diego Adiel Sandoval Chávez (principal), Aida Yarira Reyes } \\
\text { Escalante (igual), Luz Elena Tarango Hernández (apoya), Luz } \\
\text { Elena Terrazas Mata (apoya). }\end{array}$ \\
\hline $\begin{array}{l}\text { Escritura - Preparación del } \\
\text { borrador original }\end{array}$ & $\begin{array}{l}\text { Diego Adiel Sandoval Chávez (principal), Ana Córdova y } \\
\text { Vázquez (apoya), Aida Yarira Reyes Escalante (apoya). }\end{array}$ \\
\hline $\begin{array}{l}\text { Escritura - Revisión y } \\
\text { edición }\end{array}$ & $\begin{array}{l}\text { Aida Yarira Reyes Escalante (principal), Ana Córdova y } \\
\text { Vázquez (apoya), Diego Adiel Sandoval Chávez (apoya). }\end{array}$ \\
\hline Visualización & $\begin{array}{l}\text { Aida Yarira Reyes Escalante (principal), Diego Adiel Sandoval } \\
\text { Chávez (apoya). }\end{array}$ \\
\hline Supervisión & $\begin{array}{l}\text { Diego Adiel Sandoval Chávez (principal), Ana Córdova y } \\
\text { Vázquez (apoya), Aida Yarira Reyes Escalante (apoya). }\end{array}$ \\
\hline Administración de Proyectos & $\begin{array}{l}\text { Diego Adiel Sandoval Chávez (principal), Ana Córdova y } \\
\text { Vázquez (apoya), Aida Yarira Reyes Escalante (apoya). }\end{array}$ \\
\hline Adquisición de fondos & $\begin{array}{l}\text { Diego Adiel Sandoval Chávez (principal), Ana Córdova y } \\
\text { Vázquez (apoya), Aida Yarira Reyes Escalante (apoya). }\end{array}$ \\
\hline
\end{tabular}

